Review

\title{
Aging, Cancer and Immunity
}

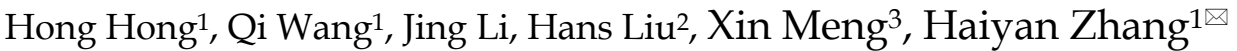 \\ 1. Department of Geriatrics, The First Hospital of China Medical University, Shenyang, 110001, China. \\ 2. Division of Geriatrics, Department of Medicine, Johns Hopkins University, Baltimore, Maryland, 21224, USA \\ 3. Department of Biochemistry and Molecular Biology, College of Basic Medical Sciences of China Medical University Shenyang, 110022, P.R. China.
}

$\triangle$ Corresponding author: Dr. Haiyan Zhang, Department of Geriatrics, the first hospital of China medical university, 155 North street Nanjing Road, Shenyang, 110001, China. Tel: +86-024-83283681; E-mail: zhanghy@cmu.edu.cn

(C) Ivyspring International Publisher. This is an open access article distributed under the terms of the Creative Commons Attribution (CC BY-NC) license (https://creativecommons.org/licenses/by-nc/4.0/). See http://ivyspring.com/terms for full terms and conditions.

Received: 2018.10.16; Accepted: 2019.04.23; Published: 2019.06.02

\begin{abstract}
Cancers are being frequently diagnosed in the elderly. Immunosenescence which refers to the gradual deterioration of the immune system brought on by natural age advancement, has been the key cross center in the increasing frequency and severity of cancer, aging and immunity. Monoclonal antibodies targeting immune checkpoint molecules CTLA-4, PD-1 or PD-LI are the promising anticancer therapeutics in multiple cancer subtypes generating remarkable and long-lasting clinical responses. These immune checkpoint blockers (ICBs)have already obtained approval for the treatment of patients with metastatic melanoma, advanced/refractory non-small cell lung cancer and renal cell cancer. ICBs can not only enhance immune responses against cancer cells but can also lead to inflammatory side effects called immune-related adverse events (irAEs). As none or only a small number of older patients were enrolled in most ICBs studies, it remains difficult to confirm the impacts of ICBs on the elderly. We could expect that clinical specificity of older patients (co-medications, comorbidities and reduced functional reserve) and immunosenescence may affect the efficacy of ICBs and tolerance in this population. However, the results from meta-analysis on the efficacy of ICBs are very encouraging and suggesting that the older patients will benefit from the ICBs revolution in oncology without increased toxicity.
\end{abstract}

Key words: Aging, Cancer, Immunity, Immunosenescence, Immunotherapy

\section{Introduction}

It is definite that the occurrence and development of many diseases, including cancers, have been shown to be associated with aging. In recent years, increasing number of researchers have come to a consensus that immune factors play more and more important roles in the process of physical degeneration and the pathologic changes, which may be the vital target for the assessment and treatment in the aged patients with tumors. To further understanding the geriatric oncology, here we provide a brief overview on the relationship between aging, cancer and immunity, besides the recent evidences of the immune management in the aged patients with tumor.

\section{Hypothesized and proven links between aging and cancer}

Aging is characterized by a progressive loss of physiological integrity, leading to impaired function.
This deterioration is the primary risk factor for major human pathologies, including cancer, cardiovascular disorders, neurodegenerative diseases and diabetes $(1,2)$. Increasing evidences have revealed the incidence of cancer augments with aging, which could be attributed to a multitude of age-associated changes including the dysregulation of the immune system (3). Advanced age is an important risk factor of cancer and is associated with poor prognosis (4). Approximately half of all malignancies are diagnosed in patients older than 65 years.

Cancer and aging can be regarded as two different manifestations of the same underlying process, specifically, the accumulation of cellular damage (1). There are several genetic or pharmacological manipulations that are capable of modulating the effects of both cancer and aging. For example, the systemic downregulation of the insulin-like growth factor 1(IGF-1) signaling pathway 
by the overexpression of PTEN tumor suppressor could increase longevity, delay aging, and confer protection against cancer on mice $(4,5)$. Similarly, the reduced expression of c-Myc oncogene could provide the elderly with resistance to several age-associated pathologies in osteoporosis, cardiac fibrosis and immunosenescence, and therefore increase their life expectancy (5).

\section{Hypothesized and proven links between aging and immunity}

\subsection{Age-associated changes in cell-mediated immunity}

Aging is a complex process that deeply affects the immune system. The decline of the immune system with age is reflected in the increased susceptibility to infectious diseases, poorer response to vaccination, increased prevalence of cancer, autoimmune and other chronic diseases.

The immune system is a complex system in which a multitude of different cells throughout the organism interact with each other, either directly or through a variety of soluble mediators, to achieve a thorough defense of the organism against foreign attacks while maintaining control of correct cell proliferation within the body. The mechanisms of the immune response have been divided into an innate and an adaptive component. The innate response comprises both the anatomical and biochemical barriers and the unspecific cellular response mediated mainly by monocytes, natural killer cells and dendritic cells. The adaptive response provides an antigen-specific response mediated by $\mathrm{T}$ and $\mathrm{B}$ lymphocytes. Both parts of the immune response are affected by the aging process.

\subsection{Immunosenescence}

Immunosenescence, which is the term given to age-associated impairments of the immune system at both cellular and serological levels, affecting the process of generating specific responses to foreign and self-antigens. There were three major theories which may explain immunosenescence, known as autoimmunity, immunodeficiency and immunodysregulation (6).

\subsubsection{The autoimmnune theory}

With increasing age, the ability of the immune system to differentiate between invaders and normal tissues diminishes. Immune cells begin to attach normal body tissues. Arthritis (7) and autoimmune thyroid disease (8) could be among the typical examples.

\subsubsection{The immune deficiency theory}

As a person ages, the immune system is no longer able to defend the body from foreign invaders and detrimental changes result.

\subsubsection{The immune dysregulation theory}

With aging, multiple changes in immune system occur disrupting the regulation between multiple components of immune process implying the progressive destruction of body cells.

Immunosenescence is a complex process that affects the immune system on the whole and reflected by the organism's capability of adequately responding to pathogens. There is no single impairment to be blamed; instead it is a multilevel dysfunction that affects individuals to a different extent. As a result, elderly people have the increased susceptibility to infections (9), decreased responses to vaccination (10) and poorer responses to known and new antigens. Additionally, aged individuals tend to present a chronic low-grade inflammatory state that has been implicated in the pathogenesis of many age-related diseases (atherosclerosis, Alzheimer's disease, osteoporosis, diabetes) (11-13).

Generally, the increased prevalence of cancer has been associated with an age-related impairment of the immune surveillance function $(14,15)$.

\section{Hypothesized and proven cellular and molecular mechanisms for aging, cancer, and immunity}

The relationship between the immune system and human cancer is dynamic and complex (16). The immune system plays a dual role in cancer development. It can not only suppress tumor growth by destroying cancer cells and inhibiting their outgrowth but also promote tumor progression either by selecting for tumor cells that are more fit to survive in an immunocompetent host or by establishing conditions within the tumor microenvironment that facilitate tumor outgrowth (17). Individual human tumors harbor a multitude of somatic gene mutations and epigenetically dysregulated genes, the products of which are potentially recognizable as foreign antigens (18). The immune system, as one of the first lines of defense, must recognize danger signals and respond accordingly (19). Immune escape and immunotolerance are considered as the main mechanism to be linked to cancer development (19-21).

Targeted immunotherapy as a potential treatment for cancer has made significant strides over the past decade based on the concept of underlying principles of tumor biology and immunology $(22,23)$. 
Cancer immunotherapy comprises a variety of treatment approaches, including antitumor monoclonal antibodies, cancer vaccines, adoptive transfer of ex vivo activated $\mathrm{T}$ and natural killer cells, and administration of antibodies or recombinant proteins that either co-stimulate immune cells or block immune inhibitory blockers (ICBs) $(16,24,25)$.

\subsection{Monoclonal antibodies}

Monoclonal antibodies (mAbs) have had a major impact on the practice of clinical oncology. The majority of mAbs approved for clinical use contain a human immunoglobulin (Ig) G1 heavy chain (16). Although much of the antitumor effect of $\mathrm{mAb}$ results from the cytotoxic effects of the drugs, it is likely that immune response also plays a role (26). The immune response and in particular antibody-dependent cell-mediated cytotoxicity (ADCC) have been proved to be the major mechanism of action via which mAbs exert their therapeutic effects. Studies in vitro, animal models, and correlative clinical investigations indicate that the interaction between $\mathrm{mAb}$ and $\mathrm{Fc}$ receptor $(\mathrm{FcR})$ contributes to the clinical antitumor activity of rituximab (26). Patients with lymphoma and a polymorphism encoding high-affinity FcR (more specifically, Fc_RIII) have a better response rate to single-agent rituximab than do patients with low-affinity $F c R(27-29)$. Cancers growing in mice lacking activating FCR fail to respond to anticancer mAbs, including rituximab and trastuzumab (30). Trastuzumab can alter human epidermal growth factor receptor 2 signaling; its ability to mediate ADCC likely also contributes significantly to its antitumor activity (31). This also applies to other mAbs that target antigens on the surface of cancer cells such as other epidermal growth factor receptor family members.

\subsection{Adoptive cell transfer}

Adoptive cell transfer (ACT) is a form of immunotherapy in which antitumor $\mathrm{T}$ cells are manipulated ex vivo and then infused into the patient. One of the examples of ACT was bone marrow transplantation (BMT) for hematologic malignancies $(32,33)$. In the 1980s, with the discovery that human $\mathrm{T}$ cells isolated from peripheral blood, tumor-draining lymph nodes, or tumor tissue could manifest selective antitumor reactivity in vitro, the cancer immunotherapy field undertook to develop specifically targeted ACT protocols.

Melanoma tumor-infiltrating lymphocytes (TILs) are a rich source of tumor-specific CD4 andCD8_Tcells relative to other malignancies (34). Autologous unfractionated TILs expanded in vitro and infused into patients with metastatic melanoma, in conjunction with systemic IL-2, have mediated objective responses in $34 \%-50 \%$ of patients $(35,36)$. Combined with more intense chemoradiotherapy preconditioning regimens, objective clinical response rates of $49 \%-72 \%$ were observed in patients with melanoma receiving highly selected TILs (37).

Chimeric antigen receptors (CARs) were engineered and used to overcome limitations of intracellular antigen processing imposed by ACT with conventional $\mathrm{T}$ cells. CARs are single-chain constructs composed of an Ig variable domain (extracellular) fused to a $\mathrm{T}$ cell receptor (TCR) constant domain; when introduced into $\mathrm{T}$ cells, they combine the antigen-recognition properties of antibodies with T-cell lytic functions, broadening the spectrum of tumor antigen recognition (38). Encouraging early clinical results with second-generation anti-CD19 CARs have been observed in patients with lymphoma $(39,40)$. However, the high affinity for target cells conferred by the Ig component of CARs, combined with amplified nonphysiologic T-cell signaling in second- and third-generation constructs, has been associated with serious adverse events (41). Reducing on-target toxicities while maintaining antitumor efficacy is an important goal of current investigations.

\subsection{Vaccine}

Long-standing interest in cancer vaccines comes from the tremendous successes of prophylactic vaccines for infectious diseases and is based on immunobiology demonstrating the capacity of $\mathrm{T}$ cells to recognize target antigens in the form of peptides complexed to surface MHC molecules. Because immunogenic peptides can be derived from proteins in every cellular compartment, essentially any protein has the potential to be recognized by $\mathrm{T}$ cells as a tumor-specific or tumor-selective antigen. Successful vaccination marshals multiple immune effector arms including CD4_and CD8_T cells to generate a potent antitumor response (42).

Despite anecdotal reports and promising phase I and II clinical trial results with cancer vaccines evaluated since the 1960s, a string of failures in randomized clinical trials has bred significant skepticism as to the ultimate clinical value of therapeutic cancer vaccines (43-45). However, in the past few years, a number of important successes with cancer vaccines have dramatically altered the perception of their potential value.

The first successful randomized phase III cancer vaccine trial used a putative dendritic cell (DC) vaccine-sipuleucel-T-to treat patients with advanced hormone-resistant prostate cancer (46). These vaccines are based on the concept that optimal T-cell activation requires antigen processing and 
presentation by a specialized cell-the DC - with the capacity to concomitantly deliver strong co-stimulatory signals in the form of membrane ligands and secreted cytokines.

Recently, two positive randomized cancer vaccine trials were reported. A melanoma vaccine consisting of a modified gp100 peptide plus systemic IL-2 was compared with systemic IL-2 alone in patients with advanced melanoma(47), yielding a statistically higher Objective response rate(ORR) in the vaccine plus IL-2 arm, improved progression free survival (PFS), and improved overall survival (OS) $(P=0.06)$. Of note, the same peptide vaccine, when combined with anti-cytotoxic T-lymphocyte antigen 4 (CTLA-4), demonstrated no improvement in patients with advanced melanomas relative to anti-CTLA-4 alone (48), underscoring the importance of context when evaluating vaccines as components of combinatorial therapies. Another trial comparing apoxvirus-prostate specific antigen prime/boost vaccine regimen plus GM-CSF versus nonantigen expressing viruses in patients with advanced prostate cancer demonstrated a significant (8 months) OS benefit for the vaccine arm but no effect on PFS or ORR (49).

With the relatively recent realization that cancer exerts animmune-tolerizing influence in the host, new trends in immunotherapy have focused on methods to interrupt tolerogenic pathways and reactivate endogenous immunity against unique as well as shared tumor antigens.

\subsection{Immune checkpoint inhibitors}

ICBs that mediate T-cell response have significantly enhanced antitumor immunity $(50,51)$. CTLA-4, also known as CD152, with its ligands CD80 and CD86, an inhibitory receptor as a global immune checkpoint engaged in priming immune responses via down-modulating the initial stages of T-cell activation, was the first clinically validated checkpoint pathway target $(25,51,52)$. Table 1 summarized the ICBs that are approved in clinic. CTLA-4 is a coinhibitory TCR, the natural function of which is to downmodulate immunity at the appropriate time, avoiding collateral normal tissue damage. Although there is no tumor specificity in the expression ofB7-1 or B7-2, potent antitumor properties of CTLA-4 blocking mAbs were nonetheless observed in preclinical models and then validated in the clinic (53). Two anti-CTLA-4 blocking mAbs-ipilimumab (Bristol-Myers Squibb, Princteon, NJ) and tremelimumab (Pfizer, New York, NY)-demonstrated similar properties in patients with advanced solid tumors in early phase clinical trials, mediating objective response rates of $10 \%$ to $15 \%$ in patients with metastatic melanoma and RCC (54-56). Ipilimumab (Yervoy; Bristol-Myers Squibb) was recently approved as first-line therapy for patients with metastatic melanoma, based on phase III trials in which this drug, administered alone or in combination with a gp100 peptide vaccine or with dacarbazine, demonstrated superior OS and PFS compared with vaccine alone (48) or dacarbazine alone (57), respectively. Approximately 20\% of patients in both studies achieved long-term survival benefit; this exceeded the reported ORRs of $10 \%$ to $15 \%$, suggesting that, as with other immunotherapies, ipilimumab may induce a state of equilibrium between the immune system and cancer, resulting in prolonged disease stabilization but not regression in some patients.

Table 1. ICBs that are approved in clinic

\begin{tabular}{|c|c|c|c|}
\hline Drugs & Pharmacy & Target & Indication \\
\hline $\begin{array}{l}\text { Opdivo } \\
\text { (Nivolumab) }\end{array}$ & BMS & $\begin{array}{l}\text { Programmed cell } \\
\text { death-1(PD-1) }\end{array}$ & $\begin{array}{l}\text { Melanoma, Non-small cell lung } \\
\text { cancer, Renal cell carcinoma, } \\
\text { Classical hodgkin lymphoma, } \\
\text { Squamous Cell Carcinoma of } \\
\text { Head and Neck, Bladder cancer, } \\
\text { Gastric cancer, Hepatocellular } \\
\text { carcinoma, Microsatellite } \\
\text { high/Mismatch repair } \\
\text { deficiency colorectal cancer }\end{array}$ \\
\hline $\begin{array}{l}\text { Keytruda } \\
\text { (Pembrolizumab) }\end{array}$ & MSD & $\begin{array}{l}\text { Programmed cell } \\
\text { death-1(PD-1) }\end{array}$ & $\begin{array}{l}\text { Melanoma, Non-small cell lung } \\
\text { cancer, Head and neck } \\
\text { squamous carcinoma, Classical } \\
\text { hodgkin lymphoma, Squamous } \\
\text { Cell Carcinoma of Head and } \\
\text { Neck, Bladder cancer, } \\
\text { Hepatocellular carcinoma, } \\
\text { Gastric cancer, Microsatellite } \\
\text { high/Mismatch repair } \\
\text { deficiency solid tumor }\end{array}$ \\
\hline $\begin{array}{l}\text { Tecentric } \\
\text { (Atezolizumab) }\end{array}$ & Roche & $\begin{array}{l}\text { Programmed cell } \\
\text { death-1(PD-1) }\end{array}$ & $\begin{array}{l}\text { Bladder cancer、Non-small cell } \\
\text { lung cancer }\end{array}$ \\
\hline $\begin{array}{l}\text { Bavencio } \\
\text { (avelumab) }\end{array}$ & Merck & $\begin{array}{l}\text { Programmed cell } \\
\text { death } \\
\text { ligand-1(PD-L1) }\end{array}$ & $\begin{array}{l}\text { Bladder cancer、 Merkel cell } \\
\text { carcinoma }\end{array}$ \\
\hline $\begin{array}{l}\text { Imfinzi } \\
\text { (durvalumab) }\end{array}$ & Astrazeneca & $\begin{array}{l}\text { Programmed cell } \\
\text { death } \\
\text { ligand-1(PD-L1) }\end{array}$ & Bladder cancer \\
\hline $\begin{array}{l}\text { Yervoy } \\
\text { (Ipilimumab) }\end{array}$ & BMS & CTLA-4 & Melanoma \\
\hline
\end{tabular}

Programmed cell death-1 (PD-1, also known as CD279) is another inhibitory receptor. Of three anti-PD-1 mAbs currently in the clinic for cancer therapy-MDX-1106/BMS936558 (Medarex, Princeton, NJ; Bristol-Myers Squibb), CT-011 (CureTech, Yavne, Israel) and MK-3475 (Merck, Whitehouse Station, NJ). A first-in-human phase I trial of intermittent dosing showed durable objective responses in 3 of 39 patients with treatment-refractory metastatic solid tumors (melanoma, RCC, and colorectal cancer), and clinical responses correlated with pretreatment expression of B7-H1/PD-L1 in the tumor (24). An ongoing trial administering MDX-1106 biweekly has shown preliminary evidence of durable objective tumor responses in approximately one third 
of patients with advanced melanoma and RCC. Of interest, objective tumor responses to MDX-1106 have also occurred in patients with refractory non-small cell lung cancer, highlighting activity against a nonimmunogenic tumor. A blocking antibody against the major ligand for PD-1/PD-L1 (MDX-1105/ BMS936559) - is also in phase I clinical trial in patients with advanced solid tumors, including melanoma, RCC, and non-small cell lung cancer.

Overall, the exciting revolution of ICBs development in oncology arouses great expectations in cancer patients.

\section{Hypothesized and proven links among aging, immunity, and cancer}

Cancer is primarily a disease of older adults (4, 58, 59). Monoclonal antibodies targeting immune checkpoint molecules CTLA-4, PD-1 or PD-L1 are emerging as promising anticancer therapeutics in multiple cancer subtypes with improved efficacy and better safety profiles when compared to traditional cytotoxic drugs (60). ICBs have already obtained approval for the treatment of patients with metastatic melanoma, advanced/refractory non-small cell lung cancer and renal cell cancer. While there are no specific trials for elderly, ICBs treatment of elderly presents a unique challenge. Comorbidities and their immune system age-related impairment might affect the function and tolerance of ICBs. Current literature does not allow us to draw definitive conclusions regarding the role of ICBs in older adults.

In 2016, a meta-analysis of ICBs randomized trials has studied efficacy of ICBs in older patients compared to young adults (61). A total of 5265 patients (ICBs: 2925; controls: 2340) were included in the analysis from three ipilimumab trials, one tremelimumab trial, four nivolumab trials and one pembrolizumab trial. The underlying malignancies included were melanoma (5 trials), non-small cell lung cancer ( 2 trials), prostate cancer ( 1 trial) and renal cell carcinoma ( 1 trial). Eight trials used 65 years and one trial used 70 years as age cut-off to conduct subgroup analyses. A total of 4725 patients from eight trials were included in the analysis of HRs for OS. The patients were dichotomized into younger and older groups with an age cut-off of 65-70 years. For younger patients, the pooled HR for OS showed significant difference between ICBs and controls (HR, 0.75; 95\% $\mathrm{CI}, 0.68-0.82 ; \mathrm{P}<0.001)$. For older patients, ICBs also significantly improved OS (HR, 0.73; 95\% CI, $0.62-0.87 ; \mathrm{P}<0.001)$ in comparison with controls. There was no statistically significant difference between subgroups of younger and older patients concerning the pooled HRs for OS $(\mathrm{P}=0.96)$.
ICBs may be responsible for specific toxicities called "immune related adverse events" (irAEs)(62-65). These irAEs are related to the infiltration of normal tissues by activated $\mathrm{T}$ cells responsible for autoimmunity. Fortunately, most of these serious immune-related adverse events are individually rare $(<1 \%)$. Immune-related side effects may be more challenging in older patients due to reduced functional reserve and age-associated comorbidities. Moreover immunosenescence could affect the efficacy and/or the toxicity of ICBs (66). Paradoxically, immunosenescence is also coupled with higher concentrations of inflammatory cytokines, called "inflammaging". Finally, older patients are known to have a higher prevalence of autoantibodies (67-69) and one can expect that ICBs may reveal subclinical autoimmune diseases.

Using ipilimumab in elderly melanoma patients, Silenireported that patients over 70 years old presented irAE with a similar frequency compared to overall population (70). Despite speculation about the specificities of older adult immunity, the current safety data appears to be similar to the population at large.

Across the different approved ICBs, no overall differences in safety were reported in elderly patients ( $\geq 65$ y.o.) and no dose adjustment is recommended $(60,71)$. The currently approved ICBs have not been evaluated in patients with severe renal or hepatic impairment. Nevertheless, no dose adjustment is recommended for patients with mild or moderate renal impairment (i.e. $\geq 30 \mathrm{ml} / \mathrm{min}$ creatinine clearance) or mild hepatic impairment (i.e. total bilirubin > upper limit normal to $1.5 \mathrm{~N}$ ).

As older patients with cancer are often taking medications for other comorbidities, it is important to note that the currently approved ICBs monoclonal antibodies are not metabolized by cytochrome P450 enzymes, therefore enzymatic competition is not expected. The use of corticosteroids may hypothetically interfere with ICBs efficacy and is recommended to avoid at baseline. Patients treated by anticoagulants or anti-aggregants must be carefully monitored in case of colitis symptoms (risk of gastrointestinal hemorrhage) or autoimmune thrombocytopenia.

In older adults, tolerance of irAEs should be carefully monitored as associated comorbidities may decompensate more easily. Moreover the use of some symptomatic treatments (such as antihistamine for pruritis) or corticosteroids may expose older patients to iatrogenic events such as diabetes worsening, mental status disturbance, hypertension and delirium.

Overall, ICBs such as anti-CTLA4 and anti PD1/PD-L1 are already part of the approved 
treatments for patients with advanced melanoma (72), non-small cell lung cancer and RCC (73). As most of ICBs studies have involved a low number of older patients it remains difficult to confirm the impact of this new therapeutics in elderly. One could expect that clinical specificity of older patients (comorbidities, co-medications, reduced functional reserve) and immunosenescence may affect ICBs efficacy and tolerance in this population. However, preliminary data of ICBs in the literatures are very encouraging and suggest that older adults will benefit from the ICBs revolution in oncology without increased toxicity.

\section{Acknowledgements}

This work was supported by National Natural Science Foundation of China (Grant number. 81301838, 81271292), and funding from the Irma and Paul Milstein Medical Asian American Partnership (MMAAP) Foundation Program for Senior Health fellow supported by the MMAAP Foundation (http://www.mmaapf.org) to Dr Haiyan Zhang, as well as the science and technology planning project of Shenyang, China (17-230-9-27).

\section{Competing Interests}

The authors have declared that no competing interest exists.

\section{References}

1. Lopez-Otin C, Blasco MA, Partridge L, et al. The hallmarks of aging. Cell. 2013;153:1194-217.

2. Chen $\mathrm{W}$, Zheng $\mathrm{R}$, Zhang $\mathrm{S}$, et al. Cancer incidence and mortality in China in 2013: an analysis based on urbanization level. Chin J Cancer Res. 2017;29:1-10.

3. Tomihara K, Curiel TJ, Zhang B. Optimization of immunotherapy in elderly cancer patients. Crit Rev Oncog. 2013;18:573-83.

4. Kendal WS. Dying with cancer: the influence of age, comorbidity, and cancer site. Cancer. 2008;112:1354-62.

5. Hofmann JW, Zhao X, De Cecco M, et al. Reduced expression of MYC increases longevity and enhances healthspan. Cell. 2015;160:477-88.

6. Castelo-Branco C, Soveral I. The immune system and aging: a review. Gynecol Endocrinol. 2014;30:16-22.

7. Zgibor JC, Ye L, Boudreau RM, et al. Community-Based Healthy Aging Interventions for Older Adults with Arthritis and Multimorbidity. J Community Health. 2017;42:390-9.

8. Dilas LT, Icin T, Paro JN, et al. [Autoimmune thyroid disease and other non-endocrine autoimmune diseases]. Med Pregl. 2011;64:183-7.

9. Gavazzi G, Herrmann F, Krause KH. Aging and infectious diseases in the developing world. Clin Infect Dis. 2004;39:83-91.

10. Sasaki S, Sullivan M, Narvaez $C F$, et al. Limited efficacy of inactivated influenza vaccine in elderly individuals is associated with decreased production of vaccine-specific antibodies. J Clin Invest. 2011;121:3109-19.

11. Griffin WS. Inflammation and neurodegenerative diseases. Am J Clin Nutr. 2006;83:470s-4s.

12. Pradhan AD, Manson JE, Rifai N, et al. C-reactive protein, interleukin 6, and risk of developing type 2 diabetes mellitus. Jama. 2001;286:327-34.

13. Lio D, Candore G, Crivello A, et al. Opposite effects of interleukin 10 common gene polymorphisms in cardiovascular diseases and in successful ageing: genetic background of male centenarians is protective against coronary heart disease. J Med Genet. 2004;41:790-4.

14. Camous X, Pera A, Solana R, et al. NK cells in healthy aging and age-associated diseases. J Biomed Biotechnol. 2012;2012:195956.

15. Grulich AE, Jin F, Poynten IM, et al. HIV, cancer, and aging. Sex Health. 2011;8:521-5

16. Topalian SL, Weiner GJ, Pardoll DM. Cancer immunotherapy comes of age. J Clin Oncol. 2011;29:4828-36.
17. Schreiber RD, Old LJ, Smyth MJ. Cancer immunoediting: integrating immunity's roles in cancer suppression and promotion. Science. 2011;331:1565-70.

18. Segal NH, Parsons DW, Peggs KS, et al. Epitope landscape in breast and colorectal cancer. Cancer Res. 2008;68:889-92.

19. Desmet CJ, Ishii KJ. Nucleic acid sensing at the interface between innate and adaptive immunity in vaccination. Nat Rev Immunol. 2012;12:479-91.

20. Campoli M, Chang CC, Ferrone S. HLA class I antigen loss, tumor immune escape and immune selection. Vaccine. 2002;20 Suppl 4:A40-5.

21. Rolinski J, Hus I. Breaking immunotolerance of tumors: a new perspective for dendritic cell therapy. J Immunotoxicol. 2014;11:311-8.

22. Kirkwood JM, Butterfield LH, Tarhini AA, et al. Immunotherapy of cancer in 2012. CA Cancer J Clin. 2012;62:309-35.

23. Ledford H. Cancer treatment: The killer within. Nature. 2014:508:24-6.

24. Brahmer JR, Drake CG, Wollner I, et al. Phase I study of single-agent anti-programmed death-1 (MDX-1106) in refractory solid tumors: safety, clinical activity, pharmacodynamics, and immunologic correlates. J Clin Oncol. 2010;28:3167-75.

25. Brahmer JR, Tykodi SS, Chow LQ, et al. Safety and activity of anti-PD-L1 antibody in patients with advanced cancer. N Engl J Med. 2012;366:2455-65.

26. Weiner GJ. Rituximab: mechanism of action. Semin Hematol. 2010;47:115-23.

27. Dall'Ozzo S, Tartas S, Paintaud G, et al. Rituximab-dependent cytotoxicity by natural killer cells: influence of FCGR3A polymorphism on the concentration-effect relationship. Cancer Res. 2004;64:4664-9.

28. Weng WK, Levy R. Two immunoglobulin $G$ fragment $C$ receptor polymorphisms independently predict response to rituximab in patients with follicular lymphoma. J Clin Oncol. 2003;21:3940-7.

29. Treon SP, Hansen M, Branagan AR, et al. Polymorphisms in FcgammaRIIIA (CD16) receptor expression are associated with clinical response to rituximab in Waldenstrom's macroglobulinemia. J Clin Oncol. 2005;23:474-81.

30. Clynes RA, Towers TL, Presta LG, et al. Inhibitory Fc receptors modulate in vivo cytotoxicity against tumor targets. Nat Med. 2000;6:443-6.

31. Hudis CA. Trastuzumab--mechanism of action and use in clinical practice. $\mathrm{N}$ Engl J Med. 2007;357:39-51.

32. Loren AW, Porter DL. Donor leukocyte infusions for the treatment of relapsed acute leukemia after allogeneic stem cell transplantation. Bone Marrow Transplant. 2008;41:483-93.

33. Chakraverty R, Mackinnon S. Allogeneic transplantation for lymphoma. J Clin Oncol. 2011;29:1855-63.

34. Topalian SL, Solomon D, Avis FP, et al. Immunotherapy of patients with advanced cancer using tumor-infiltrating lymphocytes and recombinant interleukin-2: a pilot study. J Clin Oncol. 1988;6:839-53.

35. Rosenberg SA, Yannelli JR, Yang JC, et al. Treatment of patients with metastatic melanoma with autologous tumor-infiltrating lymphocytes and interleukin 2. J Natl Cancer Inst. 1994:86:1159-66.

36. Besser MJ, Shapira-Frommer R, Treves AJ, et al. Clinical responses in a phase II study using adoptive transfer of short-term cultured tumor infiltration lymphocytes in metastatic melanoma patients. Clin Cancer Res. 2010;16:2646-55.

37. Dudley ME, Yang JC, Sherry R, et al. Adoptive cell therapy for patients with metastatic melanoma: evaluation of intensive myeloablative chemoradiation preparative regimens. J Clin Oncol. 2008;26:5233-9.

38. Gross G, Waks T, Eshhar Z. Expression of immunoglobulin-T-cell receptor chimeric molecules as functional receptors with antibody-type specificity. Proc Natl Acad Sci U S A. 1989;86:10024-8.

39. Kochenderfer JN, Wilson WH, Janik JE, et al. Eradication of B-lineage cells and regression of lymphoma in a patient treated with autologous $\mathrm{T}$ cells genetically engineered to recognize CD19. Blood. 2010;116:4099-102.

40. Kohn DB, Dotti G, Brentjens R, et al. CARs on Track in the Clinic: Workshop of the Blood and Marrow Transplant Clinical Trials Network Subcommittee on Cell and Gene Therapy Washington DC, 18 May 2010. Mol Ther. 2011;19:432-8.

41. Vandendriessche $\mathrm{T}$, Chuah MK. Xenogeneic liver models for gene therapy. Hum Gene Ther. 2010;21:1-4.

42. Pardoll DM, Topalian SL. The role of CD4+ $\mathrm{T}$ cell responses in antitumor immunity. Curr Opin Immunol. 1998;10:588-94.

43. Rosenberg SA, Yang JC, Restifo NP. Cancer immunotherapy: moving beyond current vaccines. Nat Med. 2004;10:909-15.

44. Kirkwood JM, Ibrahim JG, Sosman JA, et al. High-dose interferon alfa-2b significantly prolongs relapse-free and overall survival compared with the GM2-KLH/QS-21 vaccine in patients with resected stage IIB-III melanoma: results of intergroup trial E1694/S9512/C509801. J Clin Oncol. 2001;19:2370-80.

45. Kelland L. Discontinued drugs in 2005: oncology drugs. Expert Opin Investig Drugs. 2006;15:1309-18

46. Kantoff PW, Higano CS, Shore ND, et al. Sipuleucel-T immunotherapy for castration-resistant prostate cancer. N Engl J Med. 2010;363:411-22.

47. Schwartzentruber DJ, Lawson DH, Richards JM, et al. gp100 peptide vaccine and interleukin-2 in patients with advanced melanoma. $\mathrm{N}$ Engl J Med. 2011;364:2119-27.

48. Hodi FS, O'Day SJ, McDermott DF, et al. Improved survival with ipilimumab in patients with metastatic melanoma. N Engl J Med. 2010;363:711-23.

49. Kantoff PW, Schuetz TJ, Blumenstein BA, et al. Overall survival analysis of a phase II randomized controlled trial of a Poxviral-based PSA-targeted immunotherapy in metastatic castration-resistant prostate cancer. J Clin Oncol. 2010;28:1099-105. 
50. Pardoll DM. The blockade of immune checkpoints in cancer immunotherapy. Nat Rev Cancer. 2012;12:252-64.

51. Topalian SL, Taube JM, Anders RA, et al. Mechanism-driven biomarkers to guide immune checkpoint blockade in cancer therapy. Nat Rev Cancer. 2016;16:275-87.

52. Belum VR, Benhuri B, Postow MA, et al. Characterisation and management of dermatologic adverse events to agents targeting the PD-1 receptor. Eur J Cancer. 2016;60:12-25.

53. Leach DR, Krummel MF, Allison JP. Enhancement of antitumor immunity by CTLA-4 blockade. Science. 1996;271:1734-6.

54. Phan GQ, Yang JC, Sherry RM, et al. Cancer regression and autoimmunity induced by cytotoxic T lymphocyte-associated antigen 4 blockade in patients with metastatic melanoma. Proc Natl Acad Sci U S A. 2003;100:8372-7.

55. Yang JC, Hughes M, Kammula U, et al. Ipilimumab (anti-CTLA4 antibody) causes regression of metastatic renal cell cancer associated with enteritis and hypophysitis. J Immunother. 2007;30:825-30.

56. Ribas A. Clinical development of the anti-CTLA-4 antibody tremelimumab. Semin Oncol. 2010;37:450-4.

57. Robert C, Thomas L, Bondarenko I, et al. Ipilimumab plus dacarbazine for previously untreated metastatic melanoma. N Engl J Med. 2011;364:2517-26.

58. Elias R, Morales J, Rehman Y, et al. Immune Checkpoint Inhibitors in Older Adults. Curr Oncol Rep. 2016;18:47.

59. Extermann M. Interaction between comorbidity and cancer. Cancer Control. 2007;14:13-22.

60. Helissey C, Vicier C, Champiat S. The development of immunotherapy in older adults: New treatments, new toxicities? J Geriatr Oncol. 2016;7:325-33.

61. Nishijima TF, Muss HB, Shachar SS, et al. Comparison of efficacy of immune checkpoint inhibitors (ICIs) between younger and older patients: A systematic review and meta-analysis. Cancer Treat Rev. 2016;45:30-7.

62. Kumar V, Chaudhary N, Garg M, et al. Current Diagnosis and Management of Immune Related Adverse Events (irAEs) Induced by Immune Checkpoint Inhibitor Therapy. Front Pharmacol. 2017;8:49.

63. Arnaud-Coffin P, Maillet D, Gan HK, et al. A systematic review of adverse events in randomized trials assessing immune checkpoint inhibitors. Int J Cancer. 2019; 10.1002/ijc.32132.

64. Delanoy N, Michot JM, Comont T, et al. Haematological immune-related adverse events induced by anti-PD-1 or anti-PD-L1 immunotherapy: a descriptive observational study. Int J Cancer. 2019;6:e48-e57.

65. Salem JE, Manouchehri A, Moey M, et al. Cardiovascular toxicities associated with immune checkpoint inhibitors: an observational, retrospective, pharmacovigilance study. Lancet Oncol. 2018;19:1579-89.

66. Pawelec G. Immunosenescence and cancer. Biogerontology. 2017; 18:717-21.

67. Champiat S, Lambotte O, Barreau E, et al. Management of immune checkpoint blockade dysimmune toxicities: a collaborative position paper. Ann Oncol. 2016;27:559-74.

68. Manoussakis MN, Tzioufas AG, Silis MP, et al. High prevalence of anti-cardiolipin and other autoantibodies in a healthy elderly population. Clin Exp Immunol. 1987;69:557-65.

69. Candore G, Grimaldi MP, Listi F, et al. Prevalence of non organ-specific autoantibodies in healthy centenarians. Arch Gerontol Geriatr Suppl. 2002;8:75-80.

70. Chiarion Sileni V, Pigozzo J, Ascierto PA, et al. Efficacy and safety of ipilimumab in elderly patients with pretreated advanced melanoma treated at Italian centres through the expanded access programme. J Exp Clin Cancer Res. 2014:33:30.

71. Muchnik E, Loh KP, Strawderman M, et al. Immune Checkpoint Inhibitors in Real-World Treatment of Older Adults with Non-Small Cell Lung Cancer. J Am Geriatr Soc. 2019; 10.1111/jgs.15750.

72. Kandolf Sekulovic L, Guo J, Agarwala S, et al. Access to innovative medicines for metastatic melanoma worldwide: Melanoma World Society and European Association of Dermato-oncology survey in 34 countries. Eur J Cancer. 2018;104:201-9.

73. Shen K, Cui J, Wei Y, et al. Effectiveness and safety of PD-1/PD-L1 or CTLA4 inhibitors combined with chemotherapy as a first-line treatment for lung cancer: A meta-analysis. J Thorac Dis. 2018;10:6636-52. 